\title{
Substrate Integrated Waveguide Filtering Slot Antenna for Ka-Band Satellite Communications
}

\author{
Yuanzhi Liu and Mustapha C.E. Yagoub \\ School of Electrical Engineering and Computer Science \\ University of Ottawa \\ 161, Louis Pasteur, Ottawa, Ontario, K1N6N5 \\ Canada
}

\begin{abstract}
This paper proposes a substrate integrated waveguide (SIW) filtering slot antenna. Based on four SIW cavity resonators and a slot locating on the last resonator, a filtering antenna was designed, targeting the near $20 \mathrm{GHz}$ satellite communication band. Simulated in the Ansys-HFSS commercial software, it exhibits a $10 \mathrm{~dB}$ impedance bandwidth of $1.5 \mathrm{GHz}$ and a flat gain of $5.5 \mathrm{dBi}$ in the operating frequency band. Besides, the filtering antenna has good selectivity at passband edges and features such as compact size, low profile, and low cost, making it suitable for Ka-band satellite ground terminals.
\end{abstract}

Key-Words: - Substrate integrated waveguide, filtering antenna, slot antenna, cavity resonator.

Received: February 3, 2021. Revised: March 30, 2021. Accepted: April 2, 2021. Published: April 6, 2021.

\section{Introduction}

Due to the rapid development of wireless communications, multifunctional device design has been recognized as a key technology to miniaturize the overall circuit size while improving its performance [1], [2].

Antennas and filters are two different and independent devices in communication systems. A bandpass filter is usually cascaded right after an antenna in RF frond-end systems [3]-[5]. However, these two devices are normally designed separately and then connected. In this case, the overall performance of the system might be degraded in the frequency band(s) of interest since they may not perfectly match.

A filtering antenna, which plays the double role of antenna and bandpass filter in communication systems, was proposed to handle this issue. Besides, such a multifunctional device can miniaturize the system size to some extent. Due to their advantages, plenty of filtering antennas, especially microstripbased ones [5]-[7], have been presented in recent years. However, even though microstrip topology has various good features, e.g., low cost, ease of fabrication, etc. [9], [10], its considerable loss in high frequencies [16], such as at Ka-band, might significantly degrade the expected device performance. Therefore, microstrip topology is not a very suitable candidate for designing Ka-band filtering antennas.
In this paper, a filtering slot antenna using SIW cavity resonators is proposed. SIW is a widely used technique to design microwave and millimeter-wave (mm-wave) devices and circuits due to their attractive features such as low loss, easy of integration and fabrication, etc. [12]-[15]. More importantly, unlike microstrip lines, SIWs almost do not radiate undesired power in high frequencies [17], which is desired in antenna design.

Simulated in the Ansys-HFSS commercial software [18], the proposed filtering antenna presents a passband of 19.7-21.2 GHz with $\left|S_{11}\right|<$ $10 \mathrm{~dB}$, a flat gain of $5.5 \mathrm{dBi}$, and a high efficiency of higher than $85 \%$. Furthermore, the filtering antenna has high selectivity at passband edges and some other good features such as compact size, low profile, low cost, and ease of fabrication. These good features demonstrate that the proposed filtering antenna is a good candidate for Ka-band satellite ground terminals.

This paper is organized as follows. Section II presents the configuration of the proposed filtering antenna. Then, the performance including $\left|S_{11}\right|$, radiation patterns, etc., are presented in Section III. The good features of the proposed design are also highlighted in this section. Finally, the conclusions are drawn in Section IV.

\section{Configuration of the Proposed Filtering Antenna}

The design starts with typical SIW cavity resonators, 
which has been widely used to design bandpass filters [19]. Fig. 1 shows the configuration of the proposed filtering antenna; printed on the Rogers RT/duriod 6002 laminate (dielectric constant $\varepsilon_{r}=$ 2.94 and loss tangent $\tan \delta=0.0012$ ) with a thickness of $0.508 \mathrm{~mm}$, the device has a compact size of $15 \times 26 \mathrm{~mm}^{2}$.

The filtering antenna is constructed with four SIW resonators, which provides filtering characteristics, and a $50 \Omega$ microstrip line. A slot is located on the last resonator, allowing electromagnetic wave to be radiated, thus making the device working as a radiator/antenna. Furthermore, such a configuration can be directly used as an element to design filtering antenna arrays, which can provide more directed beam, i.e., high gain.

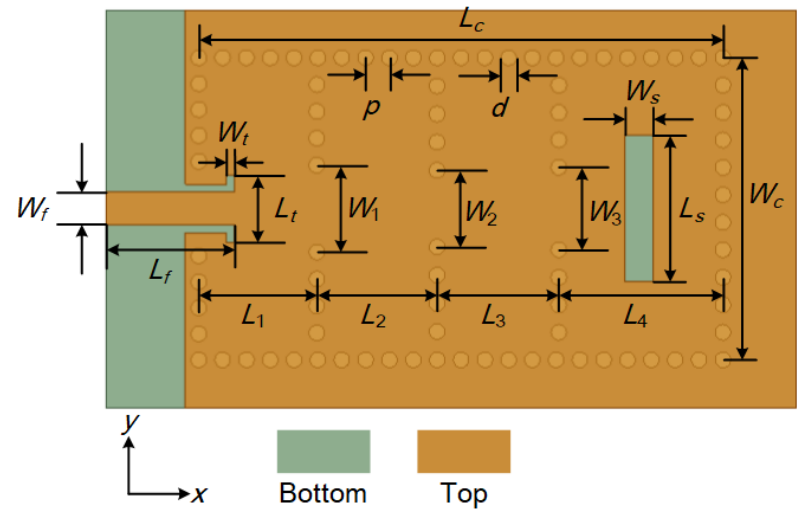

Fig. 1. Configuration of the proposed filtering antenna.

TABLE I. OPTIMIZED PARAMETERS OF THE FILTERING ANTENNA

\begin{tabular}{|c|c|c|c|}
\hline Parameter & $\begin{array}{c}\text { Value } \\
(\mathbf{m m})\end{array}$ & Parameter & $\begin{array}{c}\text { Value } \\
\mathbf{( m m )}\end{array}$ \\
\hline$W_{f}$ & 1.2300 & $L_{f}$ & 4.8391 \\
\hline$W_{c}$ & 11.3876 & $L_{c}$ & 19.7558 \\
\hline$W_{t}$ & 0.3000 & $L_{t}$ & 2.5256 \\
\hline$L_{1}$ & 4.4494 & $L_{2}$ & 4.5213 \\
\hline$L_{3}$ & 4.6000 & $L_{4}$ & 6.1851 \\
\hline$W_{s}$ & 1.0396 & $L_{s}$ & 5.5301 \\
\hline$p$ & 0.8980 & $d$ & 0.5836 \\
\hline$W_{1}$ & 3.2784 & $W_{2}$ & 2.8800 \\
\hline$W_{3}$ & 3.1092 & & \\
\hline
\end{tabular}

\section{Performance of the Filtering} Antenna

Fig. 2 shows the simulated return loss and realized gain of the filtering antenna, which illustrates its filter-like frequency response. The passband of the filtering antenna is $19.7-21.2 \mathrm{GHz}$ with $\left|S_{11}\right|<-10$ $\mathrm{dB}$ and a flat gain of $5.5 \mathrm{dBi}$. The device also exhibits good selectivity according to the obtained responses.

With the simulated gain and directivity, the efficiency of the filtering antenna was deduced. As shown in Fig. 3, the filtering antenna has an efficiency higher than $85 \%$ in the operating frequency band, mainly due to the low loss feature of the SIW cavity resonators. Out of the operating frequency band, however, the efficiency is remarkably low (lower than $10 \%$ ), which also demonstrates the filter-like performance of the antenna.

Figs. 4 and 5 show the normalized radiation of the filtering antenna at $20 \mathrm{GHz}$. The filtering antenna has typical directional radiation characteristics with half power beam width (HPBW) of $90.8^{\circ}$ and $79.0^{\circ}$ at the $x o z$ and yoz plane, respectively. Besides, the filtering antenna presents low cross polarization levels at the two planes.

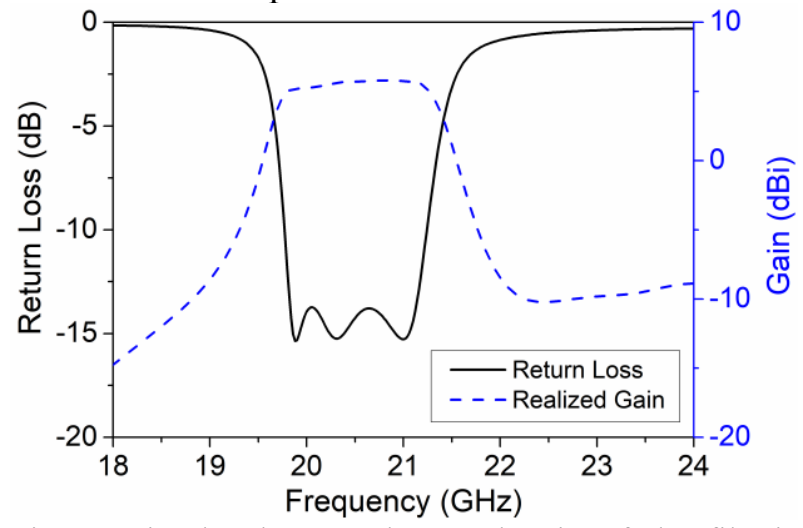

Fig. 2. Simulated return loss and gain of the filtering antenna.

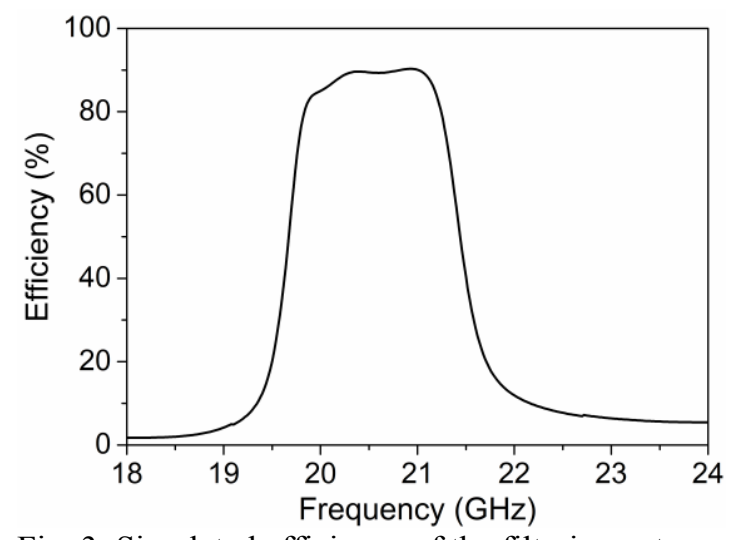

Fig. 3. Simulated efficiency of the filtering antenna.

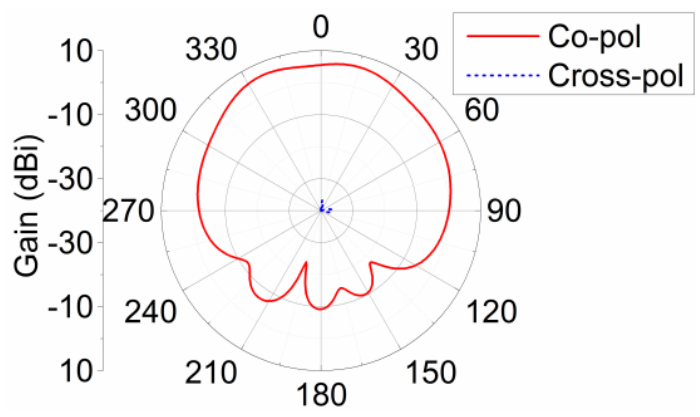

Fig. 4. Simulated radiation pattern of the filtering antenna at xoz plane. 


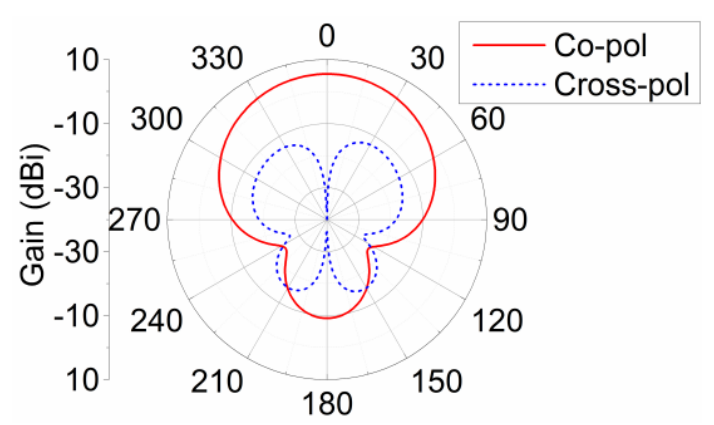

Fig. 5. Simulated radiation pattern of the filtering antenna at yoz plane.

\section{Conclusion}

In this paper, an efficient filtering antenna is proposed. Designed for Ka-band communication systems and based on four SIW cavity resonators and waveguide slot configuration, the designed device demonstrates not only filter-like frequency response both for return loss and gain, but also typical directional radiation patterns at the operating frequency band. The high selectivity achieved as well as key features such as compactness, low profile, low cost, and ease of fabrication make the proposed filtering antenna a good candidate for Kaband satellite ground terminals. Furthermore, the proposed antenna can be directly used as an element to design large filtering antenna arrays.

\section{References:}

[1] X. Chen, F. Zhao, L. Yan and W. Zhang, "A compact filtering antenna with flat gain response Within the Passband," IEEE Antennas and Wireless Propag. Lett., vol. 12, 2013, pp. 857-860.

[2] C. Chuang and S. Chung, "Synthesis and Design of a New Printed Filtering Antenna," IEEE Trans. Antennas Propagation, vol. 59, 2011, no. 3, pp. 1036-1042.

[3] C. Lin and S. Chung, "A compact filtering microstrip antenna with quasi-elliptic broadside antenna gain response," IEEE Antennas and Wireless Propag. Lett., vol. 10, 2011, pp. 381384.

[4] C. Hsieh, C. Wu, and T. Ma, "A Compact DualBand Filtering Patch Antenna Using Step Impedance Resonators," IEEE Antennas and Wireless Propag. Lett., vol. 14, 2015, pp. 10561059.

[5] G. Mansour, M. J. Lancaster, P. S. Hall, P. Gardner, and E. Nugoolcharoenlap, "Design of filtering microstrip antenna using filter synthesis approach," Prog. Electromagn. Res., vol. 145, 2014, pp. 59-67.
[6] F. Chen, H. Hu, R. Li, Q. Chu, and M. J. Lancaster, "Design of filtering microstrip antenna array with Reduced sidelobe level," IEEE Trans. on Antennas Propag., vol. 65, 2017, no. 2, pp. 903-908.

[7] C. Lin and S. Chung, "A Filtering Microstrip Antenna Array," IEEE Trans. Microwave Theory Tech., vol. 59, no. 11, 2011, pp. 28562863.

[8] L. Li and G. Liu, "A differential microstrip antenna with filtering response," IEEE Antennas and Wireless Propag. Lett., vol. 15, 2016, pp. 1983-1986.

[9] Y. Liu, G. Bai and M.C.E. Yagoub, “A 79GHz series fed microstrip patch antenna array with bandwidth enhancement and sidelobe suppression," in 2020 Int. Conf. on Radar, Antenna, Microw., Electronics, and Telecomm., 2020, pp. 155-158, Tangerang, Indonesia.

[10] Y. Liu and M. C. Yagoub, "Compact, Broadband, and Omnidirectional Antenna Array for Millimeter-Wave Communication Systems", Journal of Microwaves, Optoelectronics and Electromagnetic Applications, vol. 20, 2021, no. 2, pp. 297-306.

[11] Y. Cassivi, L. Perregrini, P. Arcioni, M. Bressan, K. Wu, and G. Conciauro, "Dispersion characteristics of substrate integrated rectangular waveguide," IEEE Microw. Wireless Compon. Lett., vol. 12, 2001, pp.333-335.

[12] X. P. Chen and K. Wu, "Substrate integrated waveguide filter with improved stopband performance for satellite ground terminal," IEEE Trans. Microw. Theory Techn., vol. 57, 2009, no. 3, pp. 674-683.

[13] J. Xu, Z. N. Chen, X. Qing, and W. Hong, "140-GHz Planar Broadband LTCC SIW Slot Antenna Array," IEEE Trans. Antennas Propag., vol. 60, 2012, pp. 3025-3028.

[14] K. Fan, Z. Hao, Q. Yuan, J. Hu, G. Q. Luo, and W. Hong, "Wideband horizontally polarized omnidirectional antenna with a conical beam for millimeter-wave applications," IEEE Trans. Antennas Propag., vol. 66, 2018, pp. 44374448.

[15] K. Sarhadi and M. Shahabadi, "Wideband substrate integrated waveguide power divider with high isolation," IET Microwaves, Antennas \& Propag., vol. 4, 2010, pp. 817-821.

[16] S. Park, D. Shin, and S. Park, "Low side-lobe substrate-integrated-waveguide antenna array using broadband unequal feeding network for millimeter-wave handset device," IEEE Trans. 
on Antennas Propag., vol. 64, 2015, pp. 923932.

[17] Y. Liu, M.C.E. Yagoub, and M. Nassor, "Omni-directional antenna array with improved gain for 5G communication systems," in 2020 IEEE USNC-CNC-URSI North American Radio Science Meeting (Joint with AP-S Symp.), 2020, pp. 33-34, Montreal, QC, Canada.

[18] Ansys ${ }^{\circledR}$ High Frequency Structure Simulator, Release 19.1.

[19] X. Chen, K. Wu, and D. Drolet, "Substrate integrated waveguide filter with improved stopband performance for satellite ground terminal," IEEE Trans. Microw. Theory Techn., vol. 57, 2009, pp. 674-683.

[20] Q. Zhang, W. Yin, S. He, and L. Wu, "Compact substrate integrated waveguide (SIW) bandpass filter with complementary split-ring resonators (CSRRs)," IEEE Microw. Wireless Compon. Lett., vol. 20, 2008, no. 8, pp. 426-428.

[21] L. Szydlowski, N. Leszczynska, A. Lamecki, and M. Mrozowski, "A substrate integrated waveguide (SIW) bandpass filter in a box configuration with frequency-dependent coupling," IEEE Microw. Wireless Compon. Lett., vol. 22, 2012, no. 11, pp. 556-558.

\section{Contribution of individual authors to the creation of a scientific article (ghostwriting policy)}

Author Contributions: Please, indicate the role and the contribution of each author:

Example

Yuanzhi Liu carried out the simulation and the optimization.

Mustapha C.E. supervises this project.

\section{Creative Commons Attribution}

\section{License 4.0 (Attribution 4.0 \\ International , CC BY 4.0)}

This article is published under the terms of the Creative Commons Attribution License 4.0 https://creativecommons.org/licenses/by/4.0/deed.en US 